\title{
A SENSOR FUSION SYSTEM IDENTIFYING COMPLEX EVENTS FOR LOCALISATION ESTIMATION
}

\author{
David Corral-Plaza $^{1}$, Olaf Reich ${ }^{2}$, Erik Hübner ${ }^{2}$, Matthias Wagner $^{2}$ and Inmaculada Medina-Bulo ${ }^{1}$ \\ ${ }^{1}$ UCASE Software Engineering Research Group, University of Cadiz \\ Avda. de la Universidad de Cádiz 10, 11519 Puerto Real, Cádiz Spain \\ ${ }^{2}$ WSN \& IoT Research Group Frankfurt University of Applied Sciences \\ Nibelungenplatz 1, 60318 Frankfurt am Main, Hessen Germany
}

\begin{abstract}
The focus of this study in progress is based on a non-GPS navigation technique which can be used in an environment without any infrastructure. Our proposed system combines a Wireless Body Area Network (WBAN) consisting of different sensors with Complex Event Processing (CEP). For the identification of complex events for independent navigation three use cases are provided.
\end{abstract}

\section{KEYWORDS}

Wireless Body Area Network, Complex Event Processing, Sensor Fusion, BLE, MEMS

\section{INTRODUCTION}

Nowadays, the navigation relies mainly on Global Position Satellite Systems (GPSS) like the American GPS, European Galileo, Chinese Beidou or Russian GLONASS. As seen recently with Galileo, which was down for over a week, these techniques are not free of errors. Additionally, it may be the case in disaster scenarios, like for example fighting fires inside buildings or when rescuing victims after an earthquake, that GPSS is not available. In these situations, it is crucial that the positions of the rescuers can be estimated in order to navigate them. One possibility is to start from a reference point and then determine the relative position and velocity of a person. This technique is standard in navigation and position estimation and named dead reckoning (Groves, 2013). In our approach we create a mobile WBAN which consecutively records sensor data about the motion of a test person. The system should then analyze the data to determine the position of the person. Furthermore, the system should detect the three different events, when the person changes the heading, walks around an obstacle, but remaining on the course or stops walking.

\section{BASIC TECHNOLOGIES}

In this section, the technologies that are lately used in the proposed architecture are described.

\subsection{IMU}

An Inertial Measurement Unit (IMU) can be categorized into the following two types. One version with a stable platform is characterized by the fact that all inertial sensors inside are placed on a space stabilized platform. This construction is based on gyroscopes. The second type of IMU is the Strapdown-IMU. It is based on Micro-Electro-Mechanical Systems (MEMS) semiconductor sensors and signal processing with low long-term stability. Due to their size and low manufacturing costs, they are well suited for commercial applications (Titterton and Weston, 2004). The IMU is a mayor part of a dead reckoning system and operates completely independent. (Groves, 2013). 


\subsection{BLE}

WBANs have some serious advantages compared to wired ones. For example, the sensors can be arranged in a flexible manner all over the body without concerning about the cabling. Moreover, the single point of failure of accidently loosening a cable is eliminated. In our application, we use the Bluetooth Low Energy (BLE) (802.15.1) standard in order to create a WBAN. It supports the frequency bandwidth $2.4 \mathrm{GHz}$. As BLE is efficient in power consumption compared to ZigBee (Siekkinen et al., 2012), it is well suited for our application. For establishing our BLE network we need to define different roles to our devices. The device managing and initiating multiple connections is equipped with the central role. The peripheral role is characterized by the devices which have just one connection to the device with the central role (Gomez et al., 2012). With this setup, it is easy to extend the system with more and different sensors in the future.

\subsection{CEP}

CEP is an already established technology that allows us to analyse streams of information in real time (Boubeta-Puig et al., 2015). The goal of this kind of analysis is to infer complex situations. In order to perform CEP analysis two elements are required. On the one hand, the information to be analyzed are the simple events, which represent changes of states in the real world, i.e., the temperature of a room, the location of a vehicle, the consumption of a smart device, etc. On the other hand, the rules, which are the conditions these simple events have to satisfy in order to detect the complex situations mentioned above, i.e., "if the temperature of the room doubles its value in less than 60 seconds a fire is happening". The formats of these simple input events and the way of implementing these rules will depend on the tool framework selected to do CEP analytics. Of all alternatives in order to perform CEP but we would like to highlight: Apache Flink and Esper. Flink described itself as a framework to perform stateful computations over streams of data, which results in a tool that allow us to create streams of data from multiple sources (Kafka, Cassandra, Twitter, Hadoop, etc.) and perform complex transformations and analytics over these data. Esper is a solution especially designed for CEP. In Esper, the input data will be provided through its Java or .NET API in several formats (Java Maps, POJO, Avro, etc.) and the rules will be described using Esper Pattern Language (EPL), which is a language that implements and extends SQL functionalities and applies them to the stream analytics. Regarding performance, Esper is able to process up to 500000 events per second in a desktop machine, which is a significant amount of information in most of the Internet of Things (IoT) scenarios. Choosing one framework or another will depend on the scenario and requirements of the use case.

\section{USE CASES}

In this section we are going to describe the complex situations that our analysis layer is going to the detect using CEP.

\subsection{UC1: Heading}

The first use case recognizes when the person is changing the direction of the course. It can tell if the person is heading to the left, right, forward or backward. For example, if the person alters the heading, the magnetometer will also changes its values permanently. Concurrently the path of the latitude and longitude information, gathered by the GPS-Node, will also indicate a change in the direction.

\subsection{UC2: Avoiding Obstacles}

The second use case recognizes when the person is just walking around an obstacle, but remaining on course. In this case, for example the gyroscope would show a rotation around an axis when avoiding the obstacle and then displaying the rotation back on this axis after the obstacle. The GPS-Node will also change the pathway when avoiding the obstacle and then getting back on the old path after walking past the obstacle. 


\subsection{UC3: Not Moving}

The third use case recognizes if the person does not change its geographical position. This can be recognized by the accelerometer, which should not show any acceleration at the $\mathrm{x}$ - and $\mathrm{y}$ - axes. The $\mathrm{z}$-axis will display the gravity including any changes through the activity of the person, like jumping or doing squats. In this use case the GPS-Node will not change the latitude and longitude information at all.

\section{SENSOR AND SYSTEM ARCHITECTURE}

In this section we are going to describe the sensors that integrates our proposed architecture.

\subsection{Sensor Fusion}

The test person will be equipped with six IMU-Nodes, one GPS-Node and an Apple Watch Series 4 (AW4) which has a built in A-GPS and 9-axis IMU. The sensors send their data to a Raspberry Pi Zero W (RPi) in different ways. All these sensors are attached to the person's body as shown in the figure 1 .

\subsubsection{IMU-Node}

The 9-axis IMU-Nodes (Arduino Nano 33 BLE Sense) are based on MEMS. They are located at the head, chest, left wrist, hips, thighs and calves of the test person. The sensors measure and send the data to the RPi at a time interval of $0.015 \mathrm{~s}$ via BLE. They measure following information of the environment: <barometric pressure>, <environmental temperature>, <relative humidity>, <acceleration (XYZ), gyroscope (XYZ), magnetometer $(\mathrm{XYZ})>$.

\subsubsection{GPS-Node}

The GPS-Node (SparkFun GPS Breakout - XA1110) is directly linked to the RPi via a cable and is attached to the left arm of the test person. It uses the European GNSS Galileo and the Russian GNSS GLONASS. The sensor transmits its information via an UART Interface at an interval of $1 \mathrm{~s}$ including amongst others the following information: <UTC time>, <latitude and longitude>, <number of satellites>, <Speed over the ground>.

\subsubsection{Apple Watch Series 4}

The AW4 is attached to the right wrist of the test person. It offers its data via a REST Interface to the RPi at an interval of about $0.02 \mathrm{~s}$. The GPS of the AW4 uses A-GPS. Following available measured sensor data is important for the experiment: <time>, <latitude and longitude>, <location speed>, <accelerometer (XYZ)>.

\subsection{Proposed Architecture}

In order to combine all the previously described technologies, the proposed architecture based on (Corral-Plaza et al., 2018) is illustrated in figure 1. It is structured in 3 layers: Data Sources, is composed by all the sensors that are producing data in execution time. In this layer we locate the IMU-Nodes, the GPS-Node and the AW4. Data Analysis, is composed by the technologies that will be in charge of the information collection and the analytics. This layer is composed by a RPi in which a Python script and a CEP engine are running. The raw data collected from the sensors are going to be sent to the CEP engine in order to detect the situations described in Section 3. Data Consumers, is composed of the endpoints which will use the valuable knowledge that the CEP engine generates once a new complex situation is detected. This layer comprises any kind of output sources such as databases, web or smartphone apps, et cetera.

The workflow of the proposed architecture is as follows. The sensors located in the Data Sources layers start to produce data which are going to be collected by the Python script located in the second layer. Then, these collected raw data are going to be streamed as simple events to the CEP engine. 


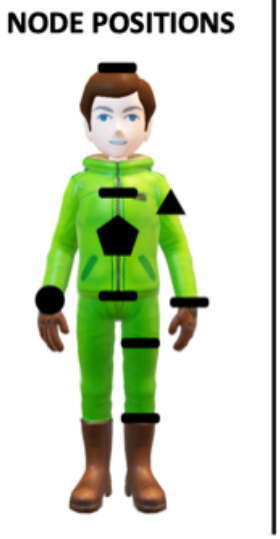

Figure 1. On the left side you can see the test person equipped with six IMUs, one GPS sensor, one AW4 and one RPi. The right side shows you the proposed architecture

Once the CEP engine receives the stream of simple events, it starts to evaluate them against the already deployed rules which describe the situations of interest that have to be detected in real time. When these conditions are satisfied, the CEP engine outputs the complex events that will be sent as alerts to the Data Consumers layer, which could be a database in order to save historical records or a smartphone app where this valuable knowledge detected by the architecture is displayed.

\section{CONCLUSION}

In this work, we have presented a novel portable architecture in order to analyse information coming from several sensors through the use of CEP capabilities. The proposed architecture would be able to gather the information of the test person in motion and, at the same time, stream these data into a CEP engine that is running within the RPi that the person wears attached to his body. Finally, CEP features are going to be used to detect situations of interest in real time, notifying them as alerts to the corresponding consumers.

\section{ACKNOWLEDGEMENT}

This work was supported in part by the Spanish Ministry of Science and Innovation and the European Union FEDER Funds (grant number TIN2015-65845-C3-3-R and RTI2018-093608-B-C33) and partly by the pre-doctoral program of the University of Cádiz (2017-020/PU/EPIF-FPI-CT/CP).

\section{REFERENCES}

Boubeta-Puig, J., Ortiz, G. and Medina-Bulo, I., 2015. MEdit4CEP: A model-driven solution for real-time decision making in SOA 2.0. Knowledge-Based Systems. Vol. 89, pp 97-112.

Corral-Plaza, D., Medina-Bulo, I., Ortiz, G. and Boubeta-Puig, J., 2018. Hacia una arquitectura para el procesamiento y análisis en tiempo real de datos heterogéneos en IoT. Actas de las XIV Jornadas de Ciencia e Ingeniería de Servicios. Presented at the JCIS 2018. Sevilla, España.

Gomez C., Oller, J. and Paradells, J., 2012. Overview and evaluation of bluetooth low energy: An emerging low-power wireless technology. In Sensors, Vol. 12, No. 9, pp 11734-11753.

Groves, P. D., 2013. Principles of GNSS, inertial, and multisensor integrated navigation systems. Artech House Publishers, Boston, USA, London, GB.

Siekkinen, M., Hiienkari, M., Nurminen, J. K. and Nieminen, J., 2012. How low energy is bluetooth low energy? Comparative measurements with zigbee/802.15.4. In IEEE wireless communications and networking conference workshops. Paris, France, pp 232-237.

Titterton, D. and Weston J., 2004. Strap-down inertial navigation technology. IET Publisher, London, GB. 